\title{
Comportamento e ciclo de vida de Sennius bondari (Pic) (Coleoptera, Chrysomelidae, Bruchinae) em Senna macranthera (Collad.) Irwin \& Barn. (Caesalpinaceae) ${ }^{1}$
}

\author{
Adelita Maria Linzmeier ${ }^{2}$, Cibele S. Ribeiro-Costa ${ }^{2} \&$ Edilson Caron ${ }^{2}$ \\ 1 Contribuição número 1472do Departamento de Zoologia, Universidade Federal do Paraná. \\ 2 Departamento de Zoologia, Universidade Federal do Paraná. Caixa Postal 19020,81531-980 Curitiba, Paraná, Brasil. \\ Bolsistas do CNPq. E-mail: alinzmeier@yahoo.com.br; stra@ufpr.br; caron@bio.ufpr.br
}

\begin{abstract}
Behavior and life cycle of Sennius bondari (Pic) (Coleoptera, Chrysomelidae, Bruchinae) in Senna macranthera (Collad.) Irwin \& Barn. (Caesalpinaceae). Some aspects of the behavior and biology of Sennius bondari (Pic, 1929) are evaluated on the host plant Senna macranthera (Collad.) Irwin \& Barn., recorded for the first time. The study was conducted on infested seeds collected in Curitiba, Paraná State. Usually eggs were deposited on the seed margin. During larval development, the cotyledons and testa are partially consumed. The pupal chamber is built inside the seed. The life cycle lasted about 140 days, with females depositing eggs from day 8 until 73. Total fecundity was slightly over 47 eggs per female, and $83 \%$ of them were viable. Adult and larval/ pupal mortality was high at $60 \%$. This may be attributed to low humidity in the lab.
\end{abstract}

KEY WORDS. Biology, damaged seeds, host plant, insects.

RESUMO. Alguns aspectos do comportamento e biologia de Sennius bondari (Pic,1929) (Coleoptera, Chrysomelidae, Bruchinae) foram avaliados em sementes de Senna macranthera (Collad.) Irwin \& Barn. (Caesalpinaceae). Esta espécie é registrada pela primeira vez como hospedeira de $S$. bondari. $O$ estudo foi realizado em sementes infestadas, coletadas em Curitiba, Paraná. Os ovos foram depositados preferencialmente na margem da semente. Durante o desenvolvimento larval, os cotilédones e a testa são parcialmente consumidos. A câmara pupal é construída dentro da semente. $O$ ciclo de vida durou cerca de 140 dias, com fêmeas depositando seus ovos do oitavo ao $73^{\circ}$ dia. A fecundidade total foi em torno de 47 ovos por fêmea e $83 \%$ destes foram viáveis. A mortalidade de adultos e larvas/pupas dentro das sementes foi alta, $60 \%$. Isso pode ser atribuído a baixa umidade no laboratório.

PALAVRAS CHAVE. Biologia, insetos, planta hospedeira, sementes danificadas.

Espécies de Sennius Bridwell, 1946 consomem principalmente sementes de leguminosas pertencentes a Senna Mill., algumas alimentam-se de Chamaecrista Moench e raramente atacam Cassia L. (Johnson 1977, 1984). Este gênero compreende 37 espécies distribuídas nas regiões Neártica e Neotropical (UDAYAGIRI \& WADHI 1989), com 18 espécies registradas para o Brasil (Silva et al. 2003).

Frutos de Senna macranthera (Collad.) Irwin \& Barn. (Caesalpinaceae) são encontrados na região urbana de Curitiba, Paraná, com sementes infestadas por Sennius bondari (Pic, 1929). Esta planta, nativa do Brasil, é muito utilizada na arborização de ruas da região sul, devido ao pequeno porte e suas características ornamentais, principalmente devido às flores amarelas (LORENZI 1992).

Sennius bondari é uma espécie generalista distribuida na Venezuela, Colômbia (Johnson 1984) e Brasil (Bahia, Rio de Janeiro, Minas Gerais, São Paulo e Paraná) (Silva et al. 1968, RibeIRO-Costa \& Reynaud 1998). Suas plantas hospedeiras encontram- se alocadas no gênero Senna: S. multijuga (Rich.) Irwin \& Barn., S. occidentalis (L.) Link, S. surattensis (Burm.) Irwin \& Barn., S. splendida (Vogel) Irwin \& Barn., S. pistaciifolia (Kunth) Irwin \& Barn., S. pendula var. advena (Vogel) Irwin \& Barn., S. bicapsularis (L.) Roxb. e S. alata (L.) Roxb. (Silva et al. 1968, Johnson 1984, MACÊDo et al. 1992, Ribeiro-Costa 1998). Bondar, em 1937, assinalou a presença de $S$. bondari em vagens de fedegoso, porém este nome popular pode se referir a várias espécies de Senna, como S. macranthera, S. alata e S. occidentalis.

O principal trabalho que indica aspectos da biologia de $S$. bondari foi o de Ribeiro-Costa (1998), que relatou a presença dessa espécie juntamente com Amblycerus submaculatus (Pic, 1927) consumindo sementes de Senna alata em Itaparica, na Bahia. Além desse trabalho, uma das principais contribuições realizadas com espécies de Sennius foi a de Center \& JoHnson (1973), que tratam de forma comparativa os aspectos bioecológicos de quatro espécies, Sennius morosus (Sharp, 1885), S. simulans (Schaeffer, 1907), S. medialis (Sharp, 1885) e $S$. fallax (Boheman, 1839). 
Por razões econômicas, a maioria dos trabalhos que trata de parâmetros biológicos e de comportamento de Bruchinae refere-se às espécies que se alimentam de sementes consumidas pelo homem, principalmente as que são pragas de grãos armazenados. Por outro lado, poucos estudos enfocam espécies que não causam danos econômicos aparentes (Johnson 1999). Dessa forma, o presente estudo visa contribuir para a redução da lacuna existente nessa área do conhecimento.

\section{MATERIAL E MÉTODOS}

Os bioensaios foram conduzidos em laboratório à temperatura média de $25,5^{\circ} \mathrm{C}, 53 \%$ U.R. e $12 \mathrm{~h}$ de fotofase. Para obtenção de exemplares de Sennius bondari (Fig. 1), foram coletadas vagens de uma árvore de Senna macranthera situada em área urbana de Curitiba, Paraná, em 16 de setembro de 2001. Em laboratório, as sementes infestadas foram isoladas em frasco de vidro $(4,5 \times 1,0 \mathrm{~cm})$ tampado com algodão, para a obtenção de indivíduos virgens. A partir dos adultos gerados, foram formados 18 casais, sexados com a observação do último esterno abdominal visível, o qual apresenta-se emarginado no macho e reto na fêmea.

Cada casal foi acondicionado em placa de Petri contendo 15 sementes de Senna macranthera não infestadas, juntamente com um pequeno recipiente contendo algodão umedecido com água e mel (10\%) para alimentação dos adultos. Os casais foram observados diariamente e o algodão trocado duas vezes por semana.

Quando as fêmeas iniciaram a oviposição, as sementes com ovos foram retiradas e isoladas em recipientes de vidro $(10,0 \mathrm{x}$ $1,5 \mathrm{~cm}$ ) com tampa de rosca, sendo substituídas por outras não infestadas, mantendo-se sempre 15 sementes. Estes recipientes foram observados diariamente até a emergência dos adultos.

Foram considerados como ovos viáveis aqueles que se apresentavam com coloração esbranquiçada, indicando que a larva de primeiro ínstar havia penetrado na semente e os inviáveis aqueles que permaneceram transparentes (Fig. 2 ov, ovn).

As sementes que possuíam a demarcação do orifício de emergência, sem que o adulto tivesse emergido, serviram para indicar a mortalidade dos adultos (Fig. 2 doe); as que continham ovos de coloração esbranquiçada e não apresentavam a demarcação do orifício de emergência do adulto, foram utilizadas para indicar a mortalidade larval/pupal.

Algumas sementes foram hidratadas para posterior dissecção, a fim de melhor compreender os aspectos relativos ao comportamento de $S$. bondari.

As análises estatísticas estão indicadas no texto, sendo os testes realizados a $\mathrm{p}<0,05$.

\section{RESULTADOS E DISCUSSÃO}

\section{Aspectos comportamentais (Figs 2-4)}

No presente estudo, considera-se pela primeira vez o re- gistro de Sennius bondari alimentando-se de sementes de Senna macranthera (Collad.) Irwin \& Barn. (Caesalpinaceae).

As vagens de $S$. macranthera são tardiamente deiscentes e as sementes dispõem-se bisseriadamente, sendo separadas por septos internos (IRWIN \& BARNEBY 1982). Ao contrário de algumas espécies de Sennius, tais como $S$. morosus, S. simulans e $S$. medialis (Center \& Johnson 1973, Ribeiro-Costa \& Costa 2002), as fêmeas de $S$. bondari (Pic, 1929) ovipositam diretamente na semente (Fig. 2), pertencendo à guilda $\mathrm{B}$ de oviposição de Johnson (1981a). O sítio preferido é a borda da semente que fica voltada para a linha ventral de união das valvas, pois é a área mais exposta durante a deiscência.

Os ovos são depositados isoladamente e, em 95,3\% das sementes, apenas um ovo foi observado. No entanto, o número de ovos por semente variou até cinco; quando ocorriam dois ou mais ovos por semente, verificou-se a emergência de apenas um adulto, indicando provavelmente competição pelo recurso alimentar. Ribeiro-Costa (1998) observou comportamento semelhante de $S$. bondari em Senna alata.

Durante o desenvolvimento, a larva alimenta-se tanto do cotilédone como da face interna do tegumento, não os destruindo completamente (Figs $3 \mathrm{co}, 4 \mathrm{acl}$ ). No último ínstar, a larva empupa no mesmo local em que os ínstares anteriores se desenvolveram, sendo os excrementos e restos de alimento compactados, formando uma câmara pupal, como descrito em Ribeiro-Costa (1998) (Fig. 3 cp). Outras espécies de Sennius que se desenvolvem em uma semente e formam o mesmo tipo de câmara pupal são S. fallax e S. medialis (CENTER \& JoHnson 1973). Johnson et al. (2001) destacaram que a maioria dos bruquíneos com tamanho pequeno, se comparadas às espécies de Amblycerus Thunberg, 1815, empupam dentro de uma única semente; é o caso da maioria das espécies de Sennius.

JoHnson (1981b) comentou que vagens com deiscência completa ao dispersarem suas sementes explosivamente dificultam o desenvolvimento das larvas de predadores pré-dispersão. Assim, Sennius bondari, como outros bruquíneos que empupam dentro da semente, encontra-se protegido após a dispersão, diminuindo o risco de morte na fase de pupa pela ação de fungos ou quaisquer outros fatores.

SANTOs et al. (1991), estudando Sennius cupreatus Kingsolver, 1987 e $S$. spodiogaster Kingsolver, 1987, em sementes de Melanoxylon brauna Scott (Fabaceae), observaram somente uma larva destes bruquíneos desenvolvendo-se por semente. Outros autores também registraram esse mesmo comportamento em duas espécies de Sennius não identificadas, predadores de sementes de Sclerolobium Vogel (Fabaceae) e em S. crudelis Ribeiro-Costa \& Reynaud, 1998, S. puncticollis (Fåhraeus, 1839) e S. nappi Ribeiro-Costa \& Reynaud, 1998, predadores de sementes de Senna multijuga (SANTos et al. 1997, L.T.SARI comunicação pessoal). Entretanto, espécies como S. laminifer (Sharp, 1885), S. morosus e $S$. simulans alimentam-se de mais de uma semente e empupam fora das mesmas (CENTER \& JoHnSON 1973, TERÁN \& L'Argentier 1979). 

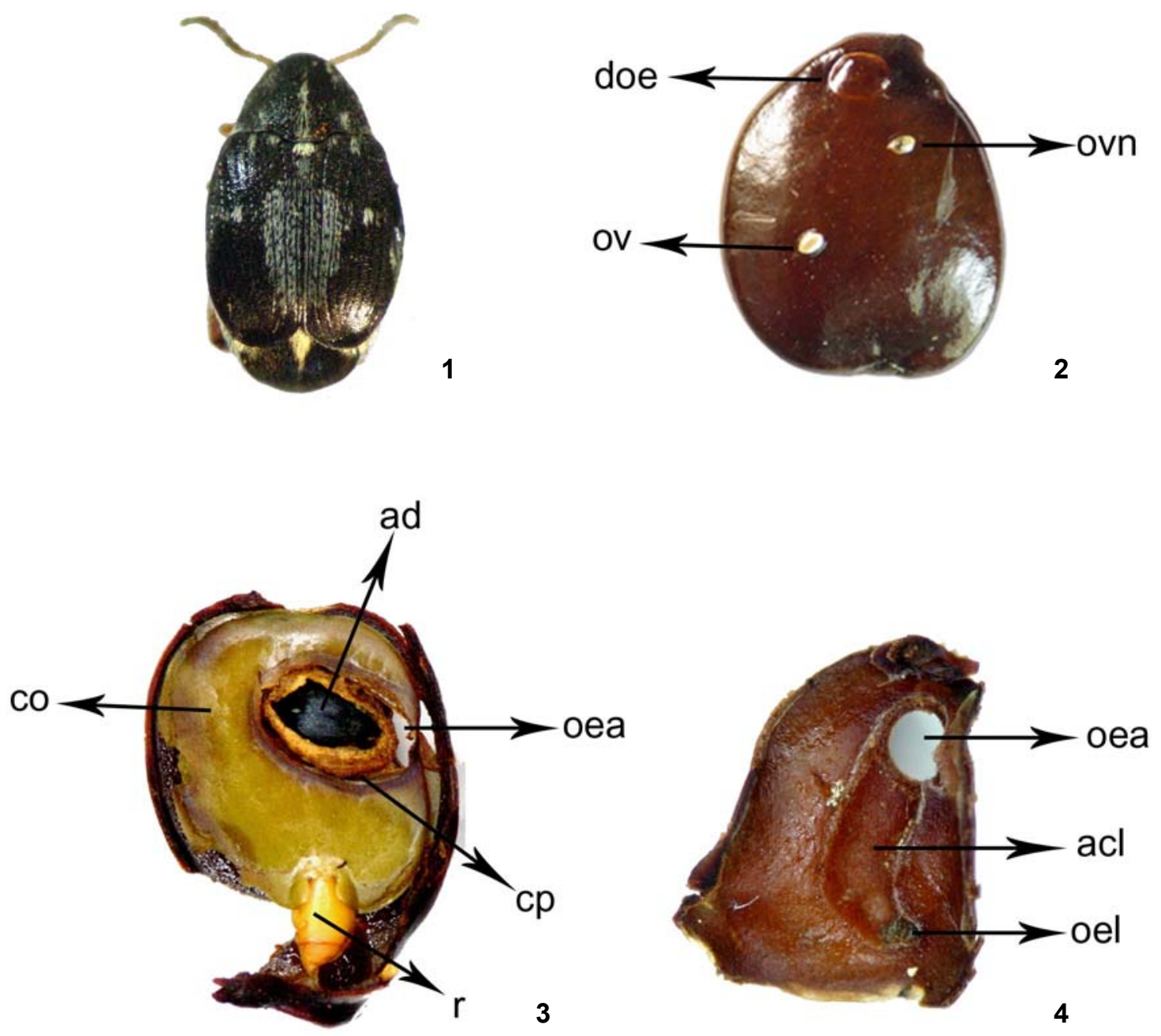

Figuras 1-4. (1) Vista dorsal de Sennius bondari, comprimento 2,5mm; (2-4) semente de Senna macranthera: (2) semente inteira; (3) semente parcialmente dissecada; (4) face interna do tegumento. (acl) Área consumida pela larva, (ad) adulto, (co) cotilédone, (cp) câmara pupal, (doe) demarcação do orifício de emergência do adulto, (oea) orifício de emergência do adulto, (oel) orifício de entrada da larva de primeiro ínstar, (ov) ovo viável, (ovn) ovo não viável, (r) radícula. Medidas: semente $6 \times 4$ mm, orifício de emergência 1,04$1,32 \mathrm{~mm}$ de diâmetro, ovo $0,68 \times 0,42 \mathrm{~mm}$.

\section{Ciclo de Vida (Tab. I, Fig. 5)}

A fecundidade e viabilidade dos ovos de Sennius bondari aproxima-se ao registrado para Zabrotes subfasciatus (Boheman, 1833), espécie-praga de Phaseolus vulgaris (SARI et al. 2003). Para $S$. bondari a média foi de $47,7 \pm 4,1$ ovos por fêmea e, no caso de $Z$. subfasciatus, o valor foi de 38,1 \pm 9 ,6. Entretanto, duas outras pragas, Acanthoscelides obtectus (Say, 1821) e Callosobruchus maculatus (F., 1775), apresentam médias superiores com 68,7 e 75,2, respectivamente (HowE \& CURRIE 1964).

Com relação à viabilidade dos ovos, o percentual foi alto, $83 \%$. Destes, cerca de 23\% deram origem a adultos. Estudos com Z. subfasciatus (Dendy \& CRedLand 1991, Cardona et al. 1989) indicaram também um alto percentual, 80\% e $90 \%$.
A duração do período de pré-oviposição foi de $8,6 \pm 0,7$ dias, variando de 6 a 13 dias, indicando que as fêmeas $S$. bondari, logo após a emergência, não estão maduras sexualmente; ao contrário, fêmeas de $Z$. subfasciatus já emergem aptas para a reprodução (Utida 1967, Pierre \& Pimbert 1981), e geralmente são capazes de copular após uma hora e ovipositar de duas a 30 horas após a cópula (PajNI \& JABBAL 1986), necessitam ainda de sementes para estimular a oogênese (Pierre \& Pimbert 1981).

O período de oviposição de $S$. bondari não é contínuo, sendo alternado por intervalos onde não ocorre oviposição, assim como registrado para $Z$. subfasciatus (Pierre \& Pimbert 1981). A média da duração desse período foi de $38,3 \pm 4,8$ dias, variando de 13 a 65 dias, bem mais longo que o registrado para 
A. M. Linzmeier et al.

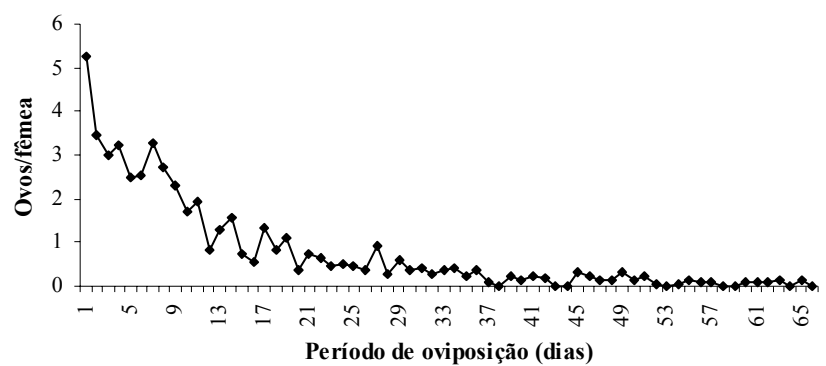

Figura 5. Número médio de ovos ao longo do período de oviposição de Sennius bondari em sementes de Senna macranthera sob condições de laboratório ( $\pm 25,5^{\circ} \mathrm{C}, 53 \%$ U.R.).

Z. subfasciatus, 5,9 $\pm 1,0$ (SARI et al. 2003) e A. obtectus, 7,9 dias (Howe \& Currie 1964). Observa-se, portanto, que a fecundidade de $S$. bondari é alta e o período de oviposição longo, diferindo das espécies-praga, em especial de $Z$. subfasciatus, que apesar de alta fecundidade apresenta curto período de oviposição. $\mathrm{O}$ período de pós-oviposição, foi de $52,6 \pm 6,2$ dias, variando de 27 a 89 dias.

A maior média de ovos postos por fêmea, 5,3, foi alcançada no primeiro dia de oviposição, decrescendo com o aumento da idade (Fig. 5). O teste não-paramétrico de Wilcoxon mostrou que há evidências de que no primeiro e segundo dia houve diferença $(p=0,06529)$. Nos dois primeiros dias foram depositados cerca de $19 \%$ do total de ovos enquanto UTIDA (1967) observou 30 a 40\% dos ovos de Z. subfasciatus logo no primeiro dia. Em geral espécies de bruquíneos-praga depositam a maior parte de seus ovos nos primeiros dias de oviposição (Howe \& CurRIE 1964).

A mortalidade total dentro da semente foi de $60 \%$, sendo $49,7 \%$ de adultos que não conseguiram emergir e 10,3\% de larvas/pupas. As causas da mortalidade não foram determinadas, entretanto o experimento foi realizado em ambiente com cerca de 53\% U.R. e isso pode ter influenciado na emergência dos adultos. Cabe salientar ainda que segundo Utida (1967), quando várias larvas desenvolvem-se em uma única semente ocorre aumento da umidade da mesma, diminuindo a mortalidade. Como 95\% das sementes apresentaram apenas um ovo, gerando então uma larva por semente, o aumento da umidade dentro da semente não seria expressivo, refletindo na mortalidade.

Howe \& CurRIE (1964) atribuíram como causas da mortalidade de $Z$. subfasciatus a incapacidade dos adultos emergirem das sementes, pela presença de uma testa espessa que impediria a entrada da larva na semente, cotilédone rígido dificultando a alimentação larval e o fato de que as larvas poderiam empupar longe da testa e, ao se transformarem em adultos, não conseguiriam emergir.

A razão sexual foi de 0,69 fêmeas para cada macho, diferentemente ao obtido para $Z$. subfasciatus desenvolvido em
Phaseolus vulgaris, onde foi em torno de 1,2 fêmeas para cada macho (Sari et al. 2003, Carvalho \& Rosseto 1968).

A longevidade média de $S$. bondari foi maior para as fêmeas, com 102,5 $\pm 2,7$ dias, do que para machos, 94,3 $\pm 5,2$ dias (Tab. I); porém, segundo o Teste U de Mann-Whitney esses valores não apresentaram diferença significativa $(p>0,10)$. Valores mais baixos de longevidade, em torno de 11 dias, foram registrados para Z. subfasciatus (Howe \& CURrIE 1964, SARI et al. 2003) e em torno de 15 dias para A. obtectus (Howe \& Currie 1964). As fêmeas de $Z$. subfasciatus, ao contrário das de $S$. bondari, tiveram maior longevidade que os machos (SARI $e t$ al. 2003), 9,4 dias para fêmeas e 13,3 para machos.

Tabela I. Duração (dias) de algumas fases do ciclo de vida e fecundidade de Sennius bondari em sementes de Senna macranthera sob condições de laboratório $\left( \pm 25,5^{\circ} \mathrm{C}, 53 \%\right.$ U.R.).

\begin{tabular}{lrrrr}
\hline \multirow{2}{*}{ Fases } & \multicolumn{4}{c}{ Adultos } \\
\cline { 2 - 5 } & Média \pm E.P. & Mínimo & Máximo & $\mathrm{n}$ \\
\hline Pré-oviposição & $8,5 \pm 0,53$ & 6 & 13 & 18 \\
Oviposição & $38,3 \pm 477$ & 13 & 65 & 16 \\
Pós-oviposição & $52,6 \pm 6,21$ & 27 & 89 & 14 \\
Longevidade da fêmea & $102,5 \pm 2,66$ & 86 & 117 & 14 \\
Longevidade do macho & $94,3 \pm 5,18$ & 41 & 121 & 16 \\
Fecundidade & $47,7 \pm 4,13$ & 23 & 75 & 16 \\
\hline
\end{tabular}

CARvalho \& Rosseto (1968) observaram que a longevidade era maior nas fêmeas que haviam colocado menor número de ovos, porém para $S$. bondari não foi observado esse padrão.

O período de desenvolvimento foi praticamente o mesmo para machos e fêmeas, $42,4 \pm 0,5$ e $42,3 \pm 0,4$ dias, respectivamente. Nos trabalhos de Howe \& Currie (1964) e Carvalho $\&$ Rosseto (1968), o período de desenvolvimento foi menor, em torno de 26 dias.

A duração do ciclo de vida foi de $136,7 \pm 5,7$ dias para os machos e $144,8 \pm 3,1$ dias para as fêmeas. Comparando-se com a duração do ciclo de vida de $Z$. subfasciatus, 28,9 \pm 8,5 dias, observa-se que o ciclo foi mais longo.

Avaliando-se os parâmetros biológicos de $S$. bondari em Senna macranthera, constataram-se algumas diferenças com relação às espécies-praga de Phaseolus vulgaris. O ciclo de vida de Sennius bondari é mais longo, bem como os períodos de préoviposição, oviposição e pós-oviposição.

\section{AGRADECIMENTOS}

Agradecemos ao Conselho Nacional de Desenvolvimento Científico e Tecnológico (CNPq), pelo auxílio concedido referente ao Proc. $N^{\circ}$ 475786/01-7, bolsas de Mestrado, Produtividade em Pesquisa e Iniciação Científica, respectivamente concedidas aos autores. 


\section{REFERÊNCIAS BIBLIOGRÁFICAS}

Bondar, G. 1937. Notas biológicas sobre bruquídeos observados no Brasil. Archivo do Instituto de Biologia Vegetal, Rio de Janeiro, 3 (1): 1-44.

Cardona, C.; C.E. Posso; J. Kornegay; J. Valor \& M. Serrano. 1989. Antibiosis effects of wild dry bean accessions on the Mexican bean weevil and the bean weevil (Coleoptera: Bruchidae). Journal of Economic Entomology, Lanham, 82: 310-315.

Carvalho, R.P.L. \& C.J. Rossetto. 1968. Biology of Zabrotes subfasciatus (Boheman) (Coleoptera: Bruchidae). Revista Brasileira de Entomologia, São Paulo, 13: 195-197.

Center, T.D. \& C.D. Johnson. 1973. Comparative life histories of Sennius (Coleoptera: Bruchidae). Environmental Entomology, Maryland, 2 (4): 669-672.

Dendy, J. \& P.F. Credland. 1991. Development, fecundity and egg dispersion of Zabrotes subfasciatus. Entomologia Experimentalis et Applicata, Dordrecht, 59: 9-17.

Howe, R.W. \& J.E. CuRrie. 1964. Some laboratory observations on the rates of development, mortality and oviposition of several species of Bruchidae breeding in stored pulses. Bulletin of Entomological Research, Oxon, 55: 437-477.

Irwin, H.S. \& R.C. BARneby. 1982. The American Cassiinae: a synoptical revision of Leguminosae, tribe Cassieae, subtribe Cassiinae in the New World. Memoirs of the New York Botanical Garden, New York, 35: 1-918.

Johnson, C.D. 1977. Life history of Ctenocolum janzeni (Coleoptera: Bruchidae) in seeds of Piscidia mollis (Leguminosae). Coleopterists Bulletin, Sacramento, 31: 313-318.

. 1981a. Interactions between bruchid (Coleoptera) feeding guilds and behavioral patterns of poods of the Leguminosae. Environmental Entomology, Maryland, 10: 249-253.

. 1981b. Seed beetle host specifity and the systematics of the Leguminosae, p. 995-1025. In: R.M. PolHill \& P.H. RAven (Eds). Advances in Leguminosae systematics. Kew, Royal Botanic Gardens, 1050p.

. 1984. Sennius yucatan, sp. nov., a redescription of $S$. infractus, and new host records for other Sennius (Coleoptera: Bruchidae). Annals of the Entomological Society of America, College Park, 77 (1): 56-64.

. 1999. Coevolution, guilds and ecology of some New World non-economic bruchid beetles, p. 91-95. In: R.C. SовтI \& J.S. YADAV (Eds). Some Aspects on the Insight of Insect Biology. Chandigarh, Panjab University, Narendra Publishing House, 316p.

Johnson, C.D.; J. Romero, \& E. Raimúndez-Urrutia. 2001. Ecology of Amblycerus crassipunctatus Ribeiro-Costa (Coleoptera: Bruchidae) in seeds of Humiriaceae, a new host family for bruchids, with an ecological comparison to other species of Amblycerus. Coleopterists Bulletin, Sacramento, 55 (1): 37-48.

LORENZI, H. 1992. Árvores brasileiras: Manual de identifica- ção de plantas arbóreas nativas do Brasil. Nova Odessa, Plantarum, 368p.

MaCÊDO, M.V.; T.M. Lewinsohn \& J.M. KIngSOLVER. 1992. New host records of some bruchid species in Brazil with the description of a new species of Caryedes (Coleoptera: Bruchidae). Coleopterists Bulletin, Sacramento, 46 (4): 330-336.

PajNI, H.R. \& A. JabBal. 1986. Some observations of Zabrotes subfasciatus (Boh.) (Bruchidae: Coleoptera). Research Bulletin of the Panjab University Science 37: 11-16.

Pierre, D. \& M. Pimbert. 1981. Some data on the reproductive activity of Zabrotes subfasciatus in the laboratory, p. 113123. In: V. Labeyrie (Ed.). The ecology of Bruchidae attacking legumes (pulses). London, W. Junk Publishers, vol. 19, 213p.

Ribeiro-Costa, C.S. 1998. Observations on the Biology of Amblycerus submaculatus (Pic) and Sennius bondari (Pic) (Coleoptera: Bruchidae) in Senna alata (L.) Roxburgh (Caesalpinaceae). Coleopterists Bulletin, Sacramento, 52 (1): 63-69.

Ribeiro-Costa, C.S. \& A.S. Costa. 2002. Comportamento de oviposição de Bruchidae (Coleoptera) predadores de sementes de Cassia leptophylla Vogel (Caesalpinaceae), morfologia dos ovos e descrição de uma nova espécie. Revista Brasileira de Zoologia, Curitiba, 19 (Supl. 1): 305-316.

Ribeiro-Costa, C.S. \& D.T. Reynaud. 1998. Bruchids from Senna multijuga (Rich) I. \& B. (Caesalpinaceae) in Brazil with descriptions of two new species. Coleopterists Bulletin, Sacramento, 52 (3): 245-252.

Santos, G.P.N.; J.C. Zanuncio; N. Anjos; J.C. Silva \& J.B. Alves. 1991. Danos causados por Sennius cupreatus e S. spodiogaster (Coleoptera: Bruchidae) em sementes de Melanoxylon braunea. Revista Ceres, Viçosa, 38: 315-322.

SANTOS, G.P.N.; T.V. ZANUnCIO; S.L.A. JÚNIOR \& J.C. ZANUNCIO. 1997. Daños por Sennius amazonicus, Sennius sp. y Amblycerus sp. (Coleoptera: Bruchidae) em semillas de Sclerobium sp. (Leguminosae). Revista de Biologia Tropical, San Jose, 45 (2): 883-886.

Sari, L.T.; C.S. Ribeiro-Costa \& P.R.V.S. Pereira. 2003. Aspectos biológicos de Zabrotes subfasciatus (Boheman, 1833) (Coleoptera, Bruchidae), em Phaseolus vulgaris L., cv. Carioca (Fabaceae), sob condições de laboratório. Revista Brasileira de Entomologia, Curitiba, 47 (4): 621-624.

Silva, A.G.; C.R.Gonçalves; D.M.Galvão; A.J.L. Gonçalves; J.Gomes; M.N. Silva \& L. Simoni. 1968. Quarto catálogo dos insetos que vivem nas plantas do Brasil, seus parasitos e predadores. Rio de Janeiro, Ministério da Agricultura, Laboratório Central de Patologia Vegetal, $1^{\circ}$ tomo, 622p.

Silva, J.A.P.; C.S. Ribeiro-Costa \& C.D. Johnson. 2003. Sennius Bridwell (Coleoptera: Bruchidae): novas espécies predadoras de sementes de Chamaecrista Moench (Caesalpinaceae) da Serra do Cipó, Minas Gerais, Brasil. Revista Brasileira de Zoologia, Curitiba, 20 (2): 269-277.

Terán, A.L. \& S.M. L'Argentier. 1979. Observaciones sobre 
Bruchidae (Coleoptera) del noroeste argentino. II. Estudios morfológicos y biológicos de algunas espécies de Amblycerinae y Bruchinae. Acta Zoologica Lilloana, Tucumán, 35 (1): 435-474.

UdAYAGIRI, S. \& S.R. W ADHI. 1989. Catalog of Bruchidae. Memoirs of the American Entomological Institute, Gainesville, 45: 1-301.

UTIDA, S. 1967. Collective oviposition and larval aggregation in Zabrotes subfasciatus (Boh.) (Coleoptera, Bruchidae). Journal of Stored Products Research, Oxon, 2: 315-322.

Recebido em 05.I.2004; aceito em 05.VI.2004.

Revista Brasileira de Zoologia 21 (2): 351-356, junho 2004 